Supporting Information

\title{
Maskless patterning of biodegradable conductors by selective laser sintering of microparticle inks and its application in flexible transient electronics
}

Shuxuan Feng, Shitai Cao, Zishen Tian, Hangyu Zhu, and Desheng Kong*

College of Engineering and Applied Sciences, National Laboratory of Solid State Microstructure, Collaborative Innovation Center of Advanced Microstructures, and Jiangsu Key Laboratory of Artificial Functional Materials, Nanjing University, Nanjing 210093, China.

*Corresponding author: dskong@nju.edu.cn 


\section{Supporting Figures}
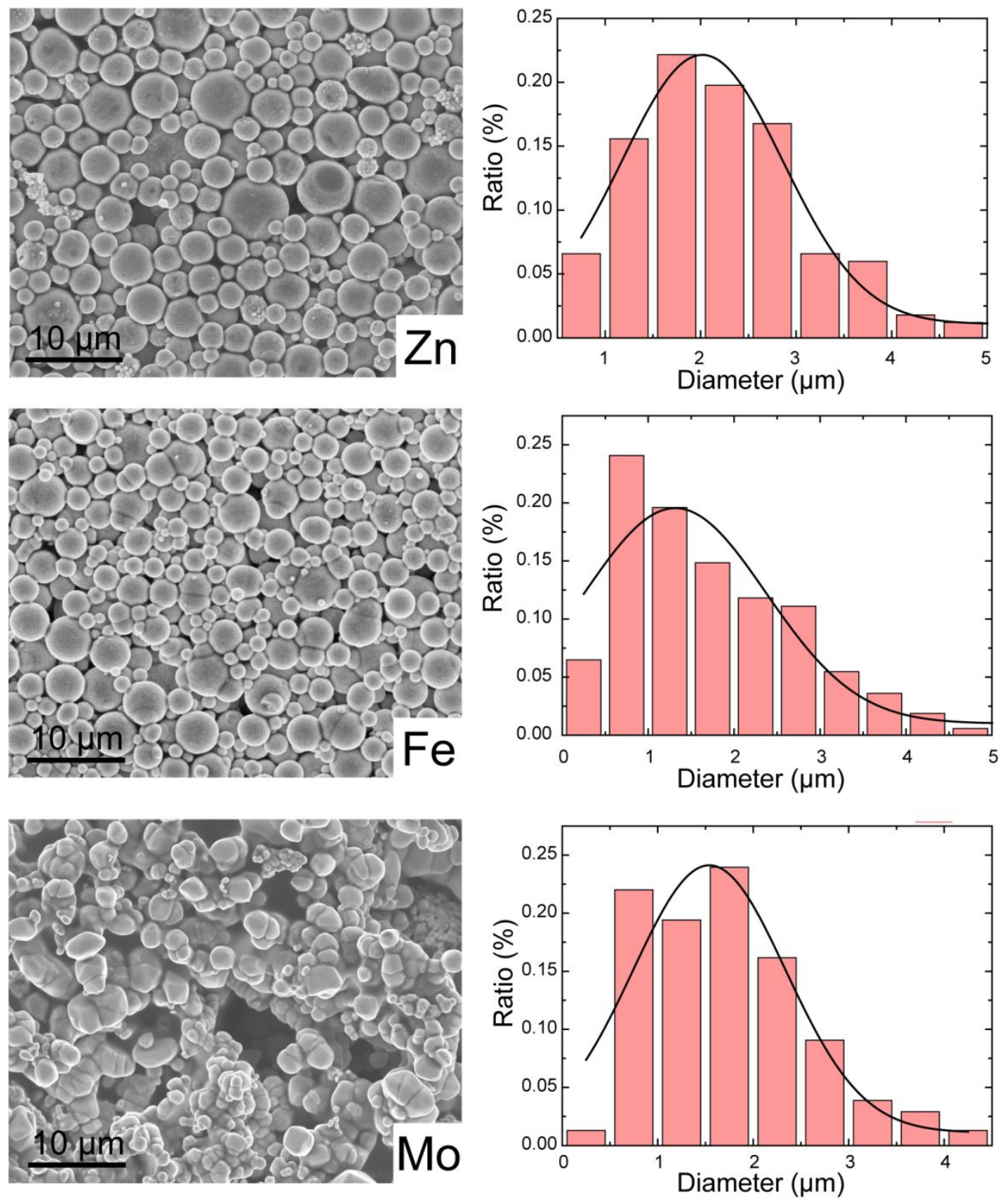

Figure S1. SEM images of biodegradable metal microparticles and corresponding histograms of size distributions. Gaussian fits to the distributions (smooth curves) yield $2.0 \pm 0.5 \mu \mathrm{m}$ for $\mathrm{Zn}$ microparticles, $1.3 \pm 0.2 \mu \mathrm{m}$ for Fe microparticles, and $1.5 \pm 0.5 \mu \mathrm{m}$ for Mo microparticles. 


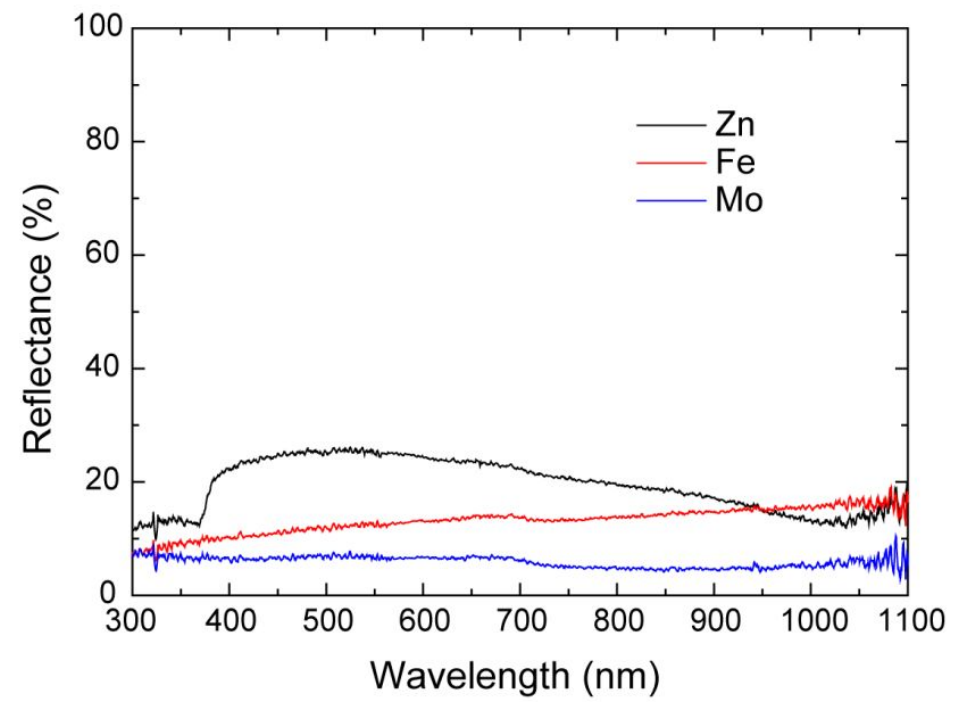

Figure S2. Reflectance spectra of spin-casted microparticle films of Zn/PVAc $(\sim 8 \mu \mathrm{m})$, $\mathrm{Fe} / \mathrm{PVAc}(\sim 10 \mu \mathrm{m})$, and Mo/PVAc $(\sim 18 \mu \mathrm{m})$ composites on PVA substrates. The low reflectance at $355 \mathrm{~nm}$, ranging from $7 \%$ to $13 \%$, is the enabler for effective sintering by UV laser.

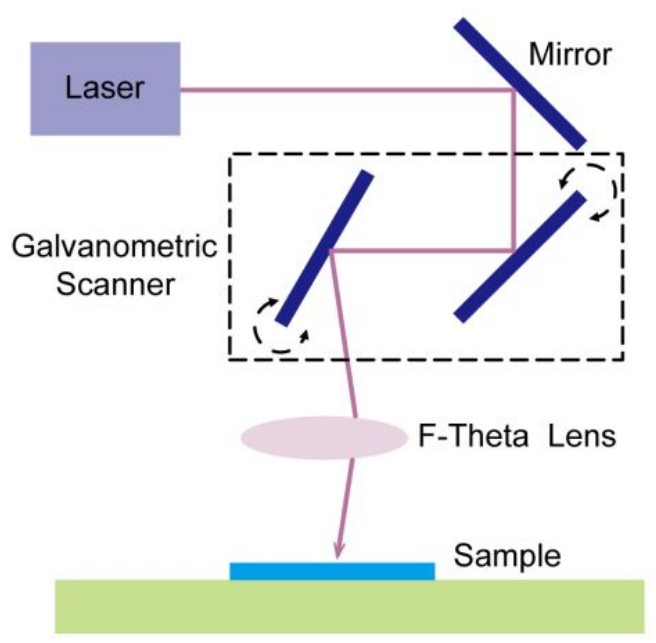

Figure S3. Schematic illustration of the laser marking system to generate arbitrary patterns according to programmed paths. 


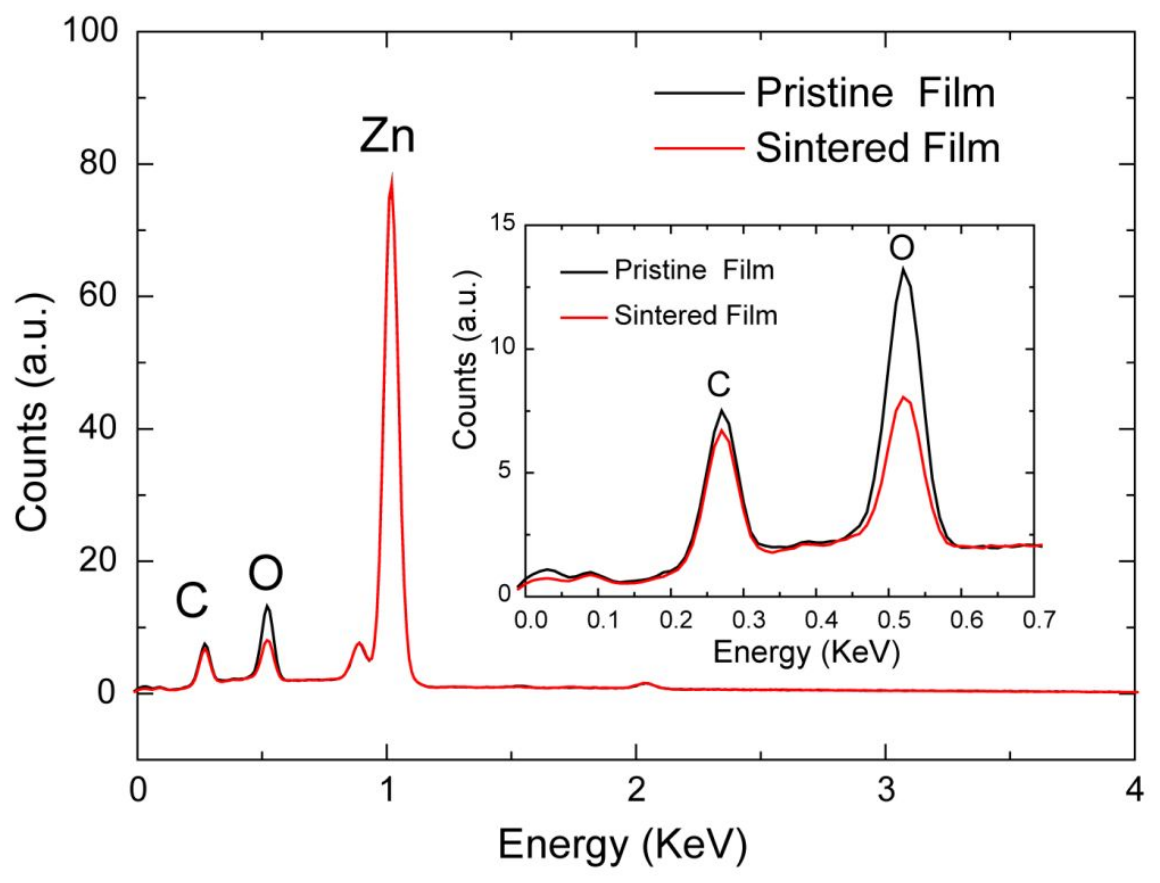

Figure S4. Energy Dispersive X-ray spectra (EDS) acquired from Zn/PVAc composite film before and after laser sintering. The spectra show decreased carbon and oxygen peaks as a result of laser treatment, which corresponds to the partial decompositions of polymeric binder of PVAc. The instantaneous laser heating likely suppresses secondary oxidation of $\mathrm{Zn}$ microparticles, which is consistent with previous experiments carried out on $\mathrm{Cu}$ nanoparticles. ${ }^{1-2}$
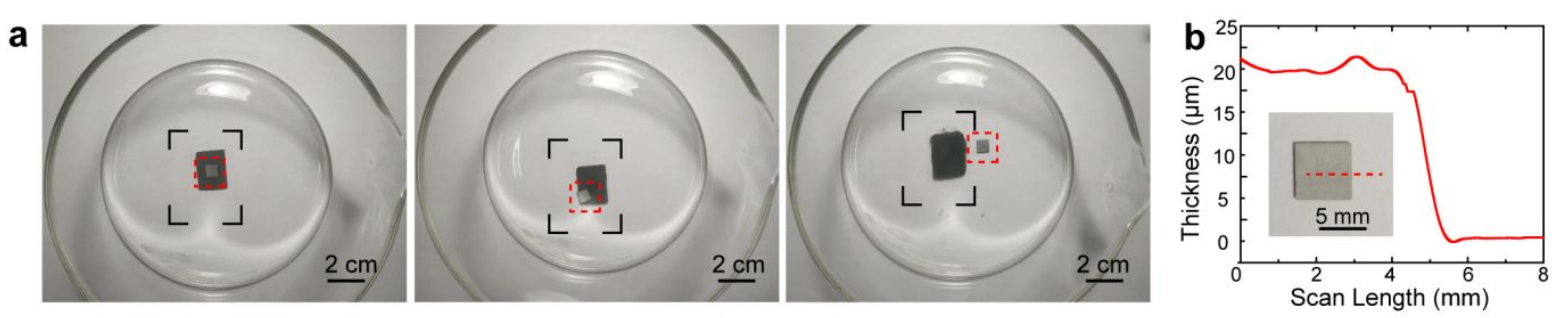

Figure S5. (a) Images showing the process to acquire fully sintered layer from printed $\mathrm{Zn} / \mathrm{PVAc}$ composites. Laser treatment converts the top fraction of $\mathrm{Zn}$ microparticles into a layer of interconnected conductor, which is retrieved as an intact film by dissolving the underlying pristine composites in ethyl acetate. (b) Height profile of fully sintered $\mathrm{Zn}$ conductor with a thickness of $\sim 20 \mu \mathrm{m}$. Inset: Corresponding image of the $\mathrm{Zn}$ conductor transferred onto a glass slide for height profile measurement. 

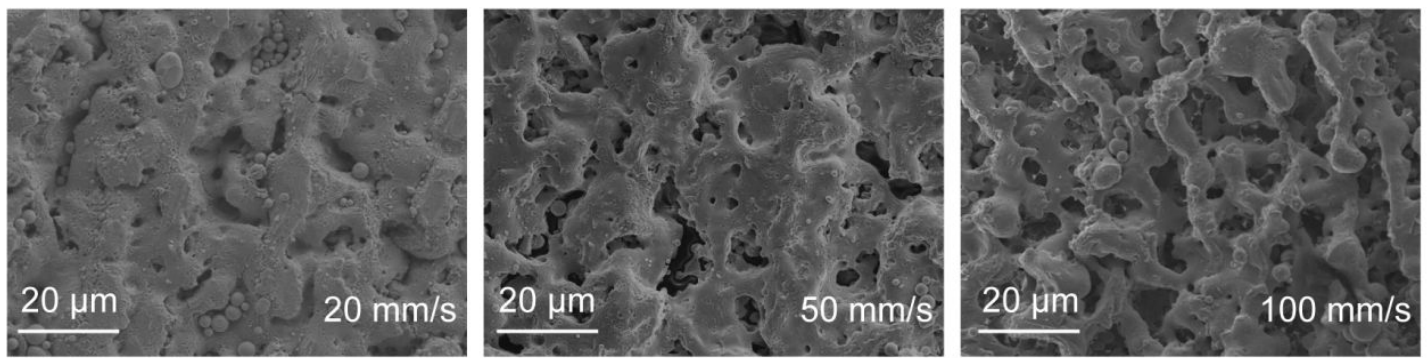

Figure S6. SEM images of sintered $\mathrm{Zn}$ conductor with varying scan speeds at a fixed laser power of $1.1 \mathrm{~W}$.

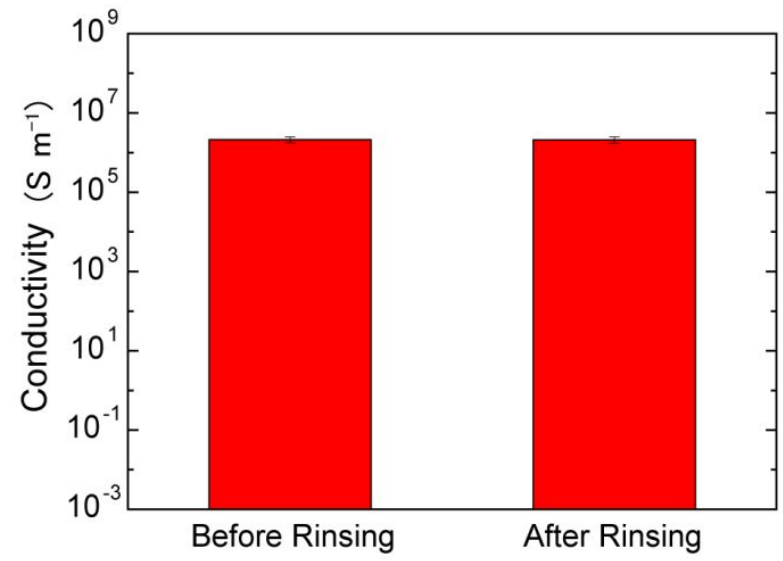

Figure S7. Conductivity of laser sintered features before and after solvent rinsing. Negligible change in conductivity verifies strong adhesion to the substrate, thereby enabling solvent rinsing for pattern development.
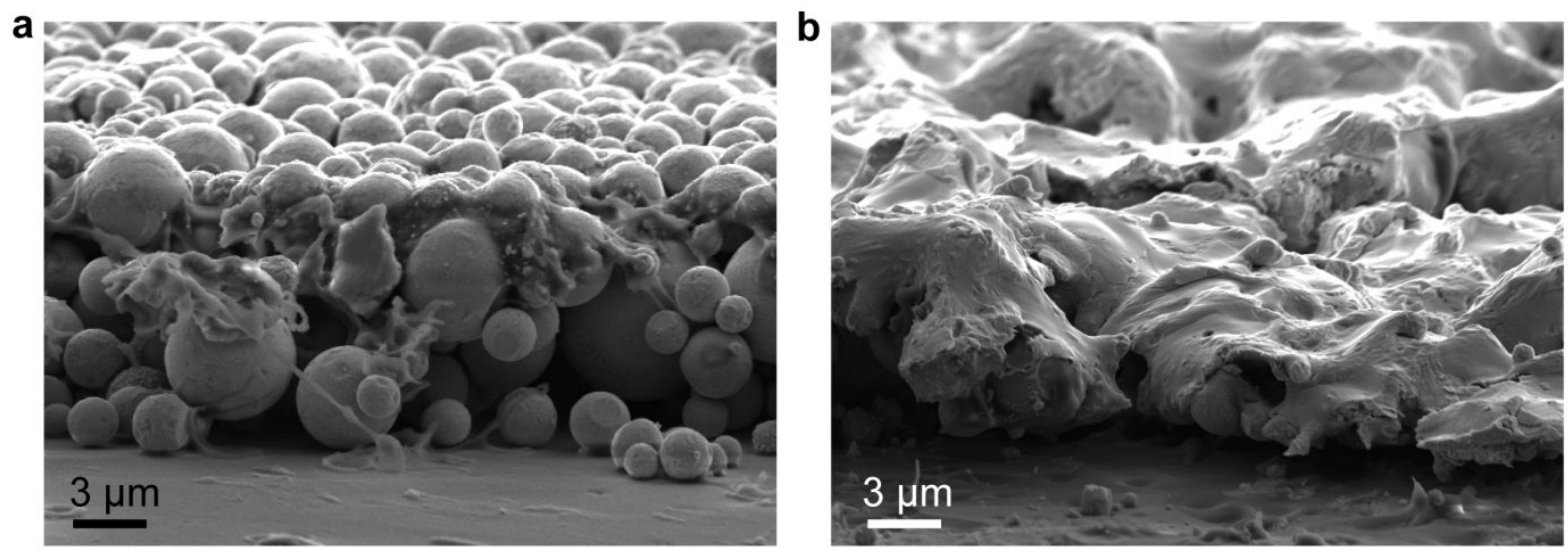
Figure S8. Cross-sectional SEM images of Zn microparticle film on PVA substrate (a) before and (b) after laser treatment. Laser sintering process produces interconnected conductor across the entire depth of the film with strong bonds to the substrate. There are not any obvious signatures of substrate damages.
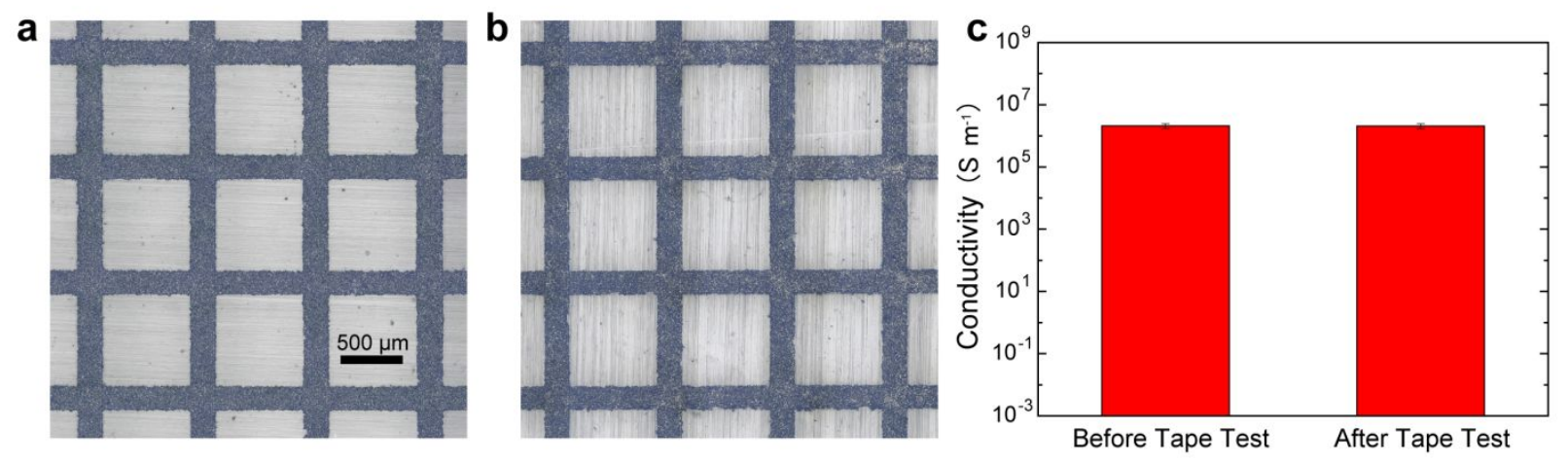

Figure S9. (a) Image of Zn conductive grid on PVA substrate. (b) Corresponding Zn grid after scotch tape test. (c) Conductivity of $\mathrm{Zn}$ conductor before and after tape test. The negligible change in conductivity confirms the excellent adhesion between the conductive features and the substrate. 

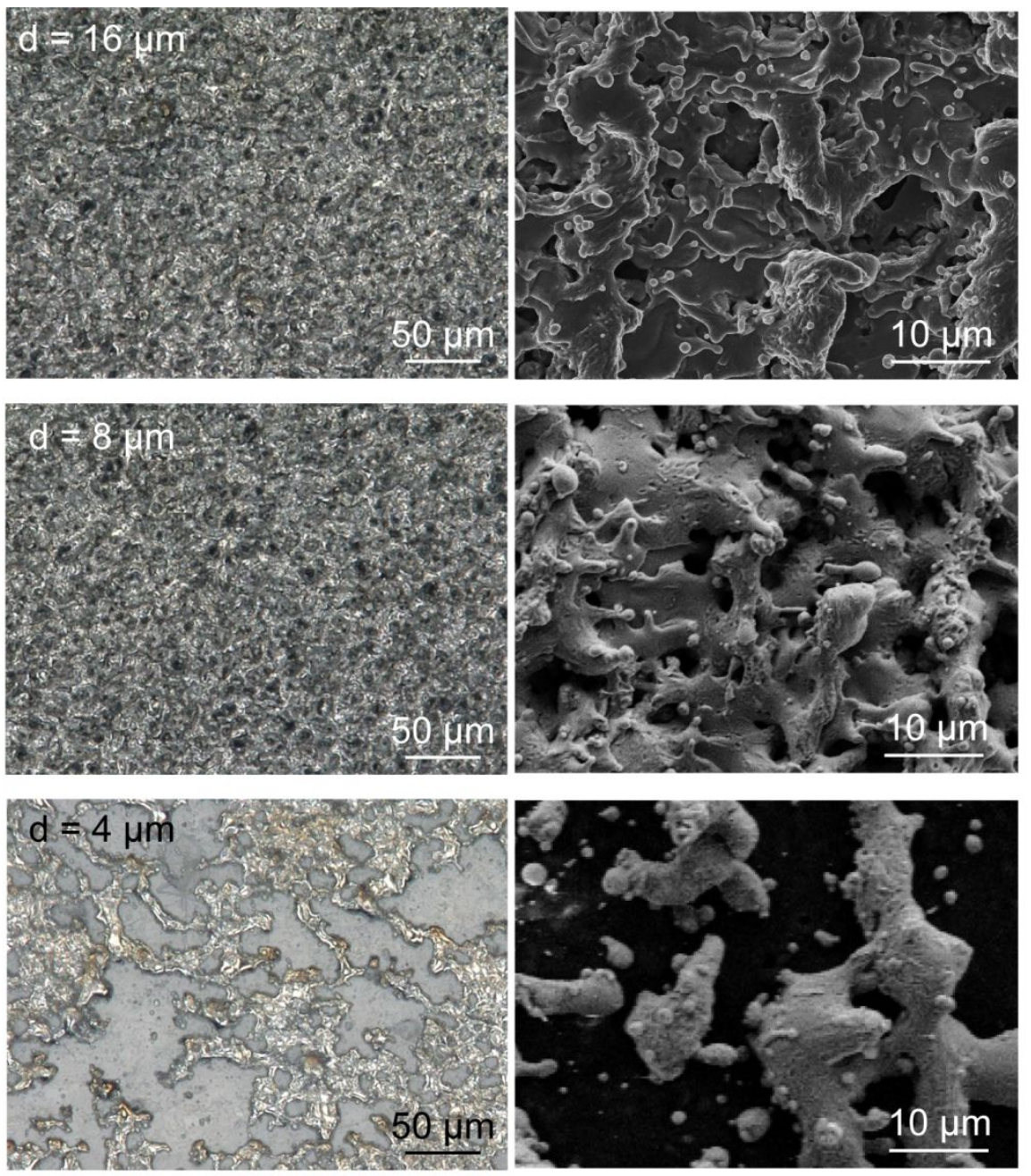

Figure S10. Optical microscope (left) and corresponding SEM (right) images of sintered Zn conductors with different thicknesses on PVA substrate. 

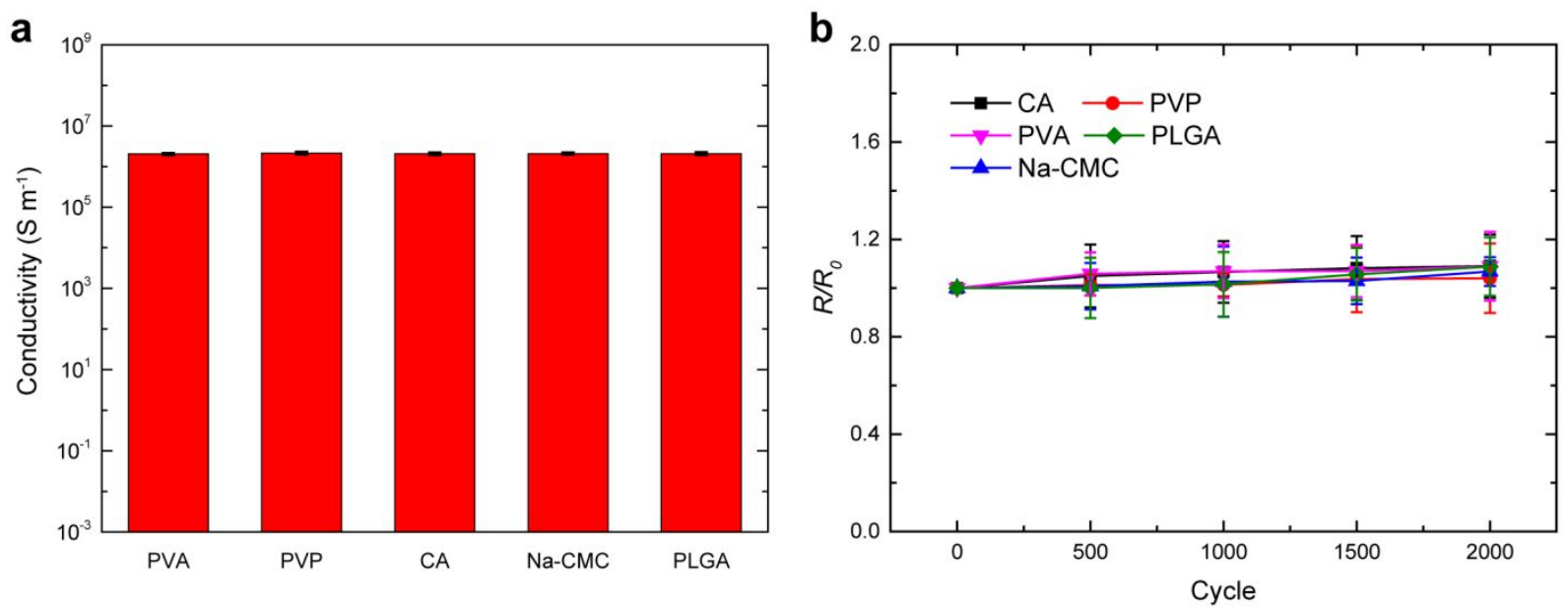

Figure S11. (a) Conductivity of $\mathrm{Zn}$ conductors created on different biodegradable substrates. (b) Evolution of the resistance for these $\mathrm{Zn}$ conductors over 2000 bending cycles to a radius of $5 \mathrm{~mm}$.
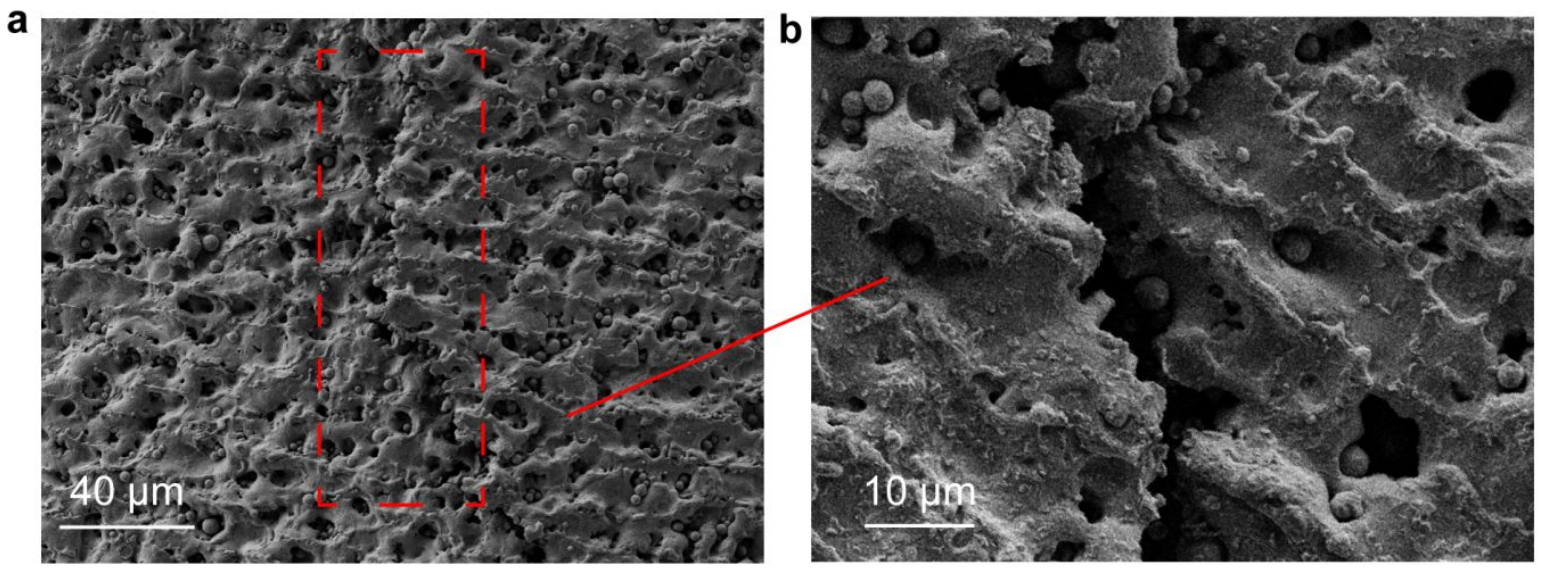

Figure S12. (a) SEM image of Zn conductor on PVA substrate after repetitive bending to 2.8 $\mathrm{mm}$ for 5000 cycles. (b) Magnified SEM image showing the presence of small cracks as the structural evidence for slightly increased resistance. 

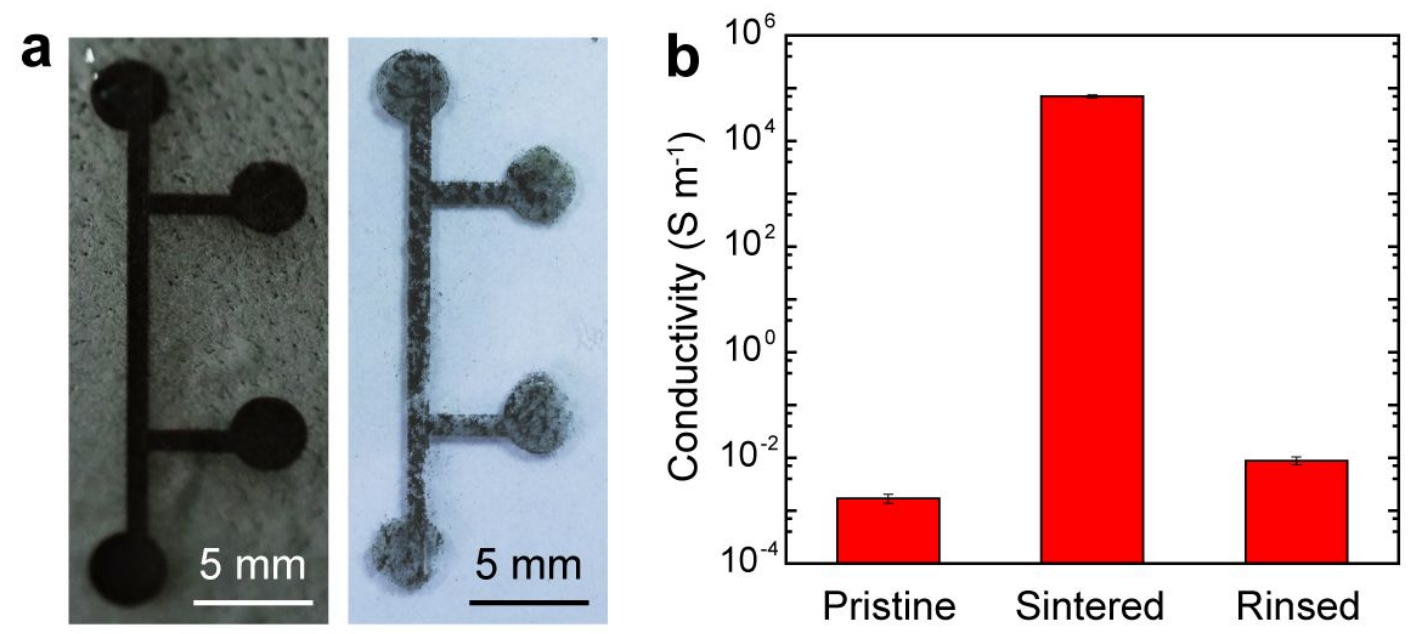

Figure S13. (a) Optical images of selectively sintered Mo patterns before and after solvent rinsing. (b) Conductivity values of Mo samples at each stage of maskless patterning process.

a

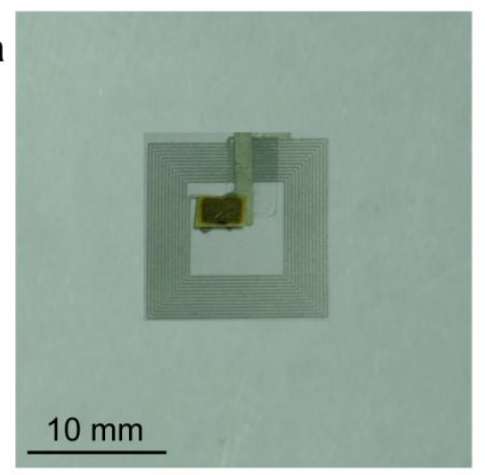

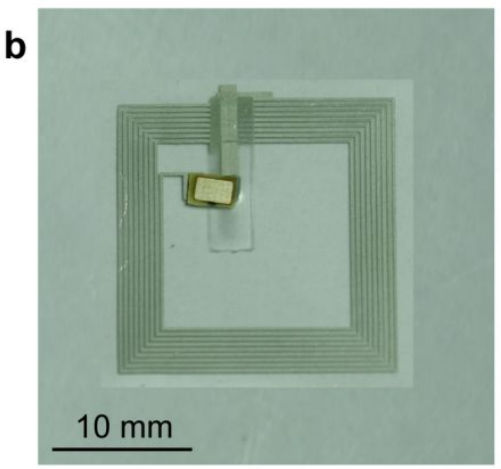

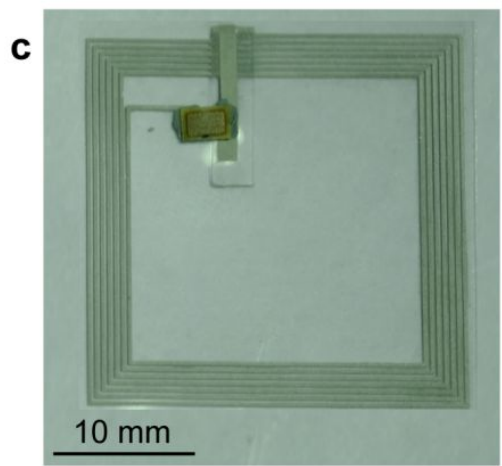

Figure S14. Optical images of NFC tags with the dimensions of (a) $13 \times 13 \mathrm{~mm}^{2}$, (b) $20 \times 20 \mathrm{~mm}^{2}$ and (c) $27 \times 27 \mathrm{~mm}^{2}$, respectively. 

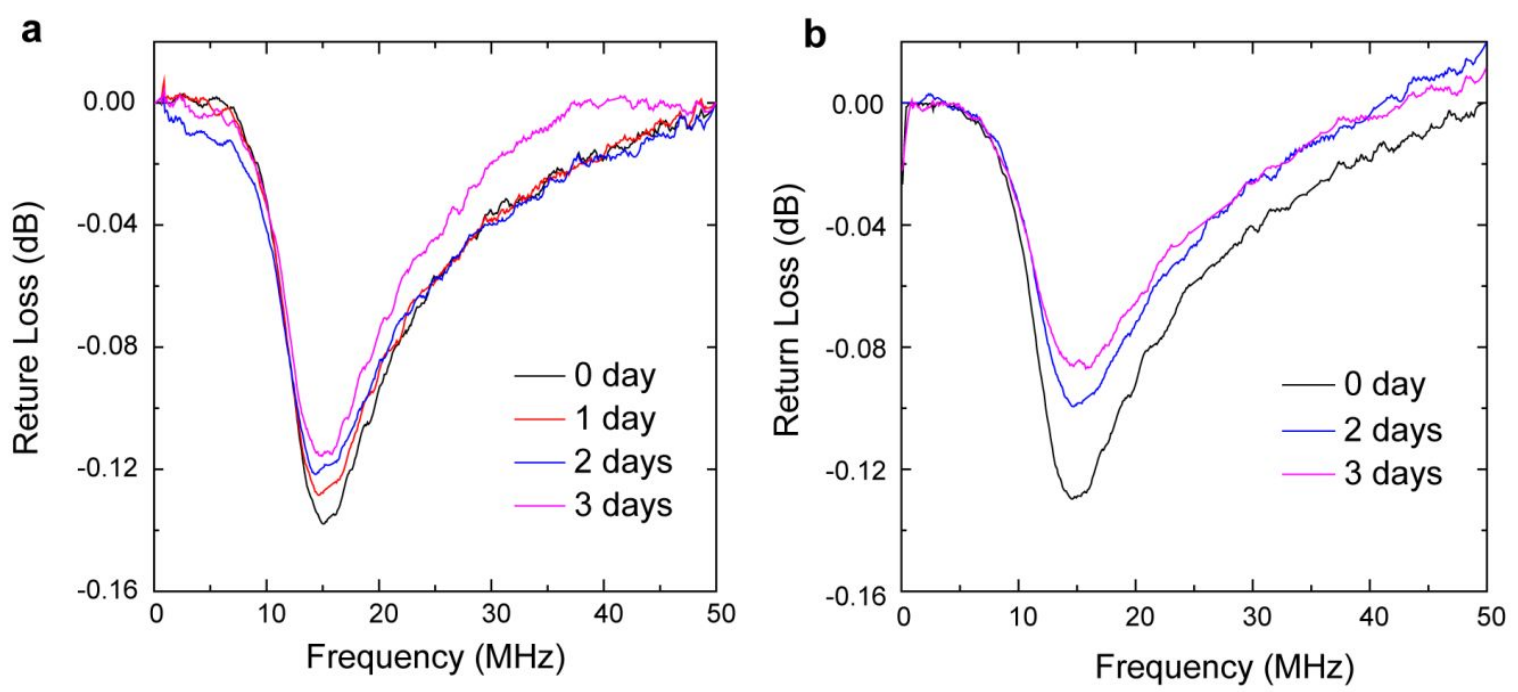

Figure S15. Change in return loss of miniaturized NFC tags $(13 \mathrm{~mm} \times 13 \mathrm{~mm})$ over 3 days storage under different relative humidity of (a) $50 \%$ and (b) $70 \%$, respectively. The absorbed energy slightly decreases with the storage time. The increased loss is largely associated with increased resistance of the antenna under high humidity levels. NFC tags remain functional for communications with smartphone after three-day storage, thereby demonstrating decent stability for operations in humid air.
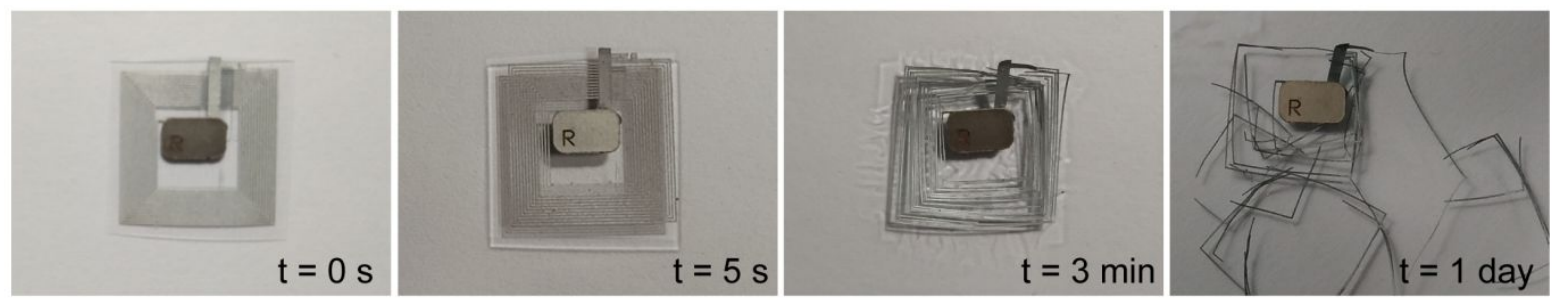

Figure S16. Images showing the disintegration process of a miniaturized NFC tag $(13 \mathrm{~mm}$ $\times 13 \mathrm{~mm}$ ) in deionized water. 

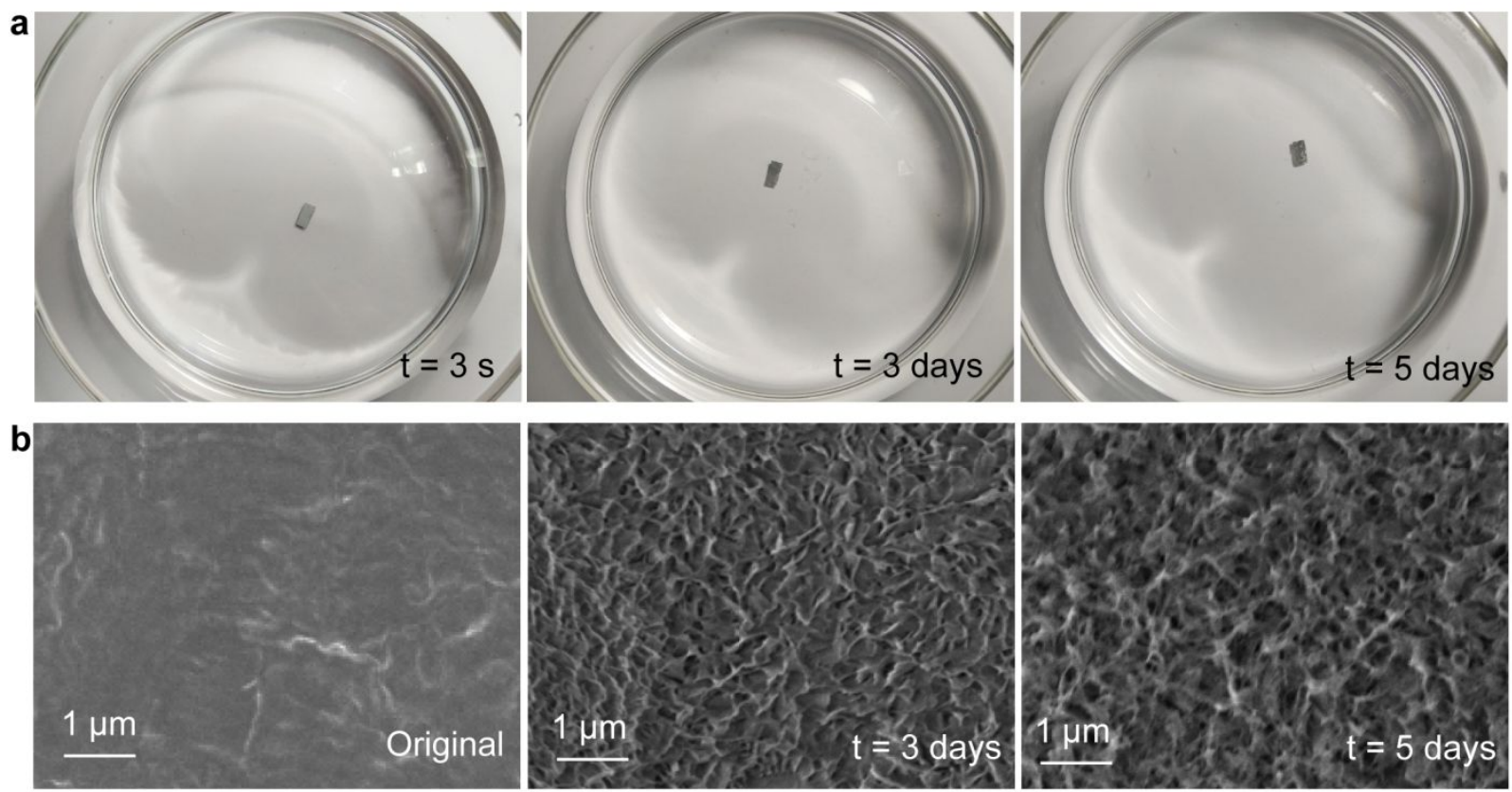

Figure S17. (a) Optical images showing the changes over 5 days for $\mathrm{Zn}$ conductor on PVA substrate in deionized water. (b) Corresponding SEM images of $\mathrm{Zn}$ conductor to reveal the microstructure evolutions as the signatures of the degradation process.

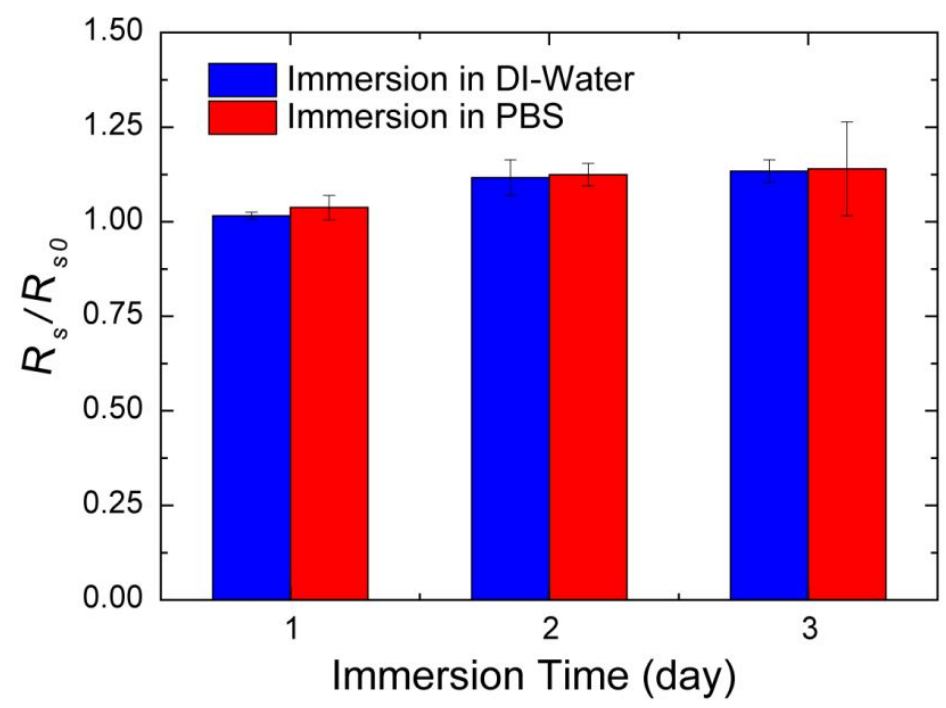

Figure S18. Change in sheet resistance of $\mathrm{Zn}$ conductor on PLGA substrate in deionized water and phosphate-buffered saline (PBS). The conductive features show minor change in resistance over three days, which demonstrate slow degradation of as-prepared conductor for short-term applications in aqueous environment. The swelling and hydrolysis of PLGA leads to the formation of cracks that prevent additional tests over extended periods. ${ }^{3-4}$ 


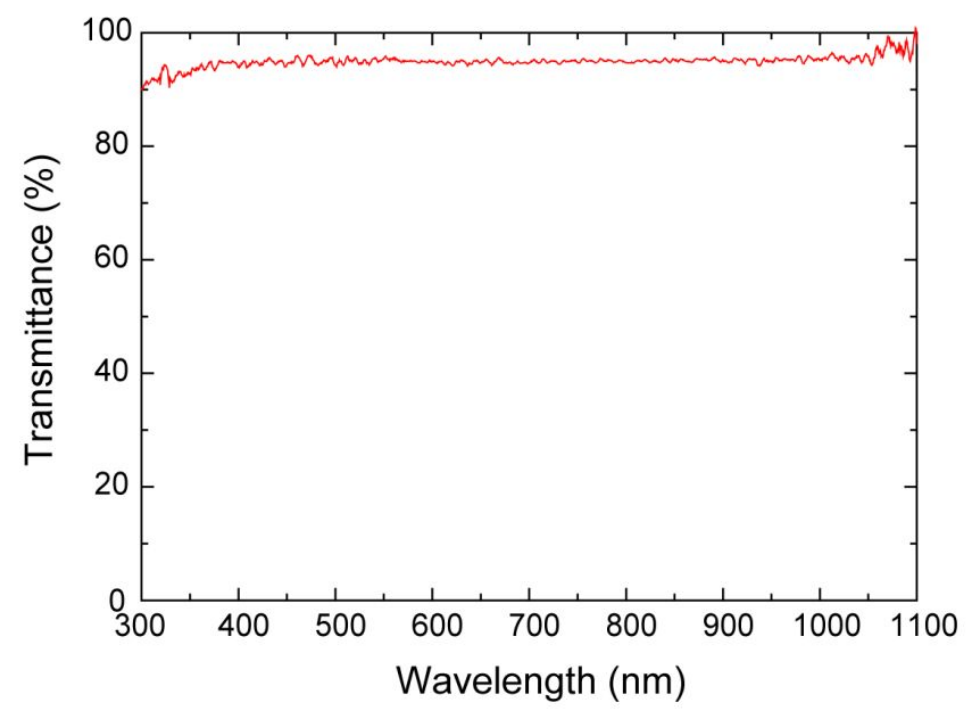

Figure S19 Transmittance spectrum of a $10 \mu \mathrm{m}$-thick PVA film. The PVA substrate is highly transparent with transmittance of $95.6 \%$ at $550 \mathrm{~nm}$.

\section{Supporting Videos}

Video S1. The operation of a miniturized NFC tag mounted on the fingernail. As a smartphone approaches the tag, the stored message "Fingernail-sized NFC" is retrieved by the smartphone running NFC TagWriter application

\section{Supporting References}

(1) Paquet, C.; James, R.; Kell, A. J.; Mozenson, O.; Ferrigno, J.; Lafrenière, S.; Malenfant, P. R. Photosintering and electrical performance of $\mathrm{CuO}$ nanoparticle inks. Org. Electron. 2014, 15 (8), 1836-1842.

(2) Rager, M. S.; Aytug, T.; Veith, G. M.; Joshi, P. Low-thermal-budget photonic processing of highly conductive $\mathrm{cu}$ interconnects based on $\mathrm{CuO}$ nanoinks: potential for flexible printed electronics. ACS Appl. Mater. Inter. 2016, 8 (3), 2441-2448.

(3) Hsu, B. B.; Park, M.-H.; Hagerman, S. R.; Hammond, P. T. Multimonth controlled small molecule release from biodegradable thin films. P. Natl. Acad. Sci. 2014, 111 (33), 1217512180. 
(4) Fredenberg, S.; Wahlgren, M.; Reslow, M.; Axelsson, A. The mechanisms of drug release in poly (lactic-co-glycolic acid)-based drug delivery systems-a review. Int. J. of pharmaceut. 2011, 415 (1-2), 34-52. 